\title{
ENERGY CASCADES AND FLUX LOCALITY IN PHYSICAL SCALES OF THE 3D NAVIER-STOKES EQUATIONS
}

\author{
R. DASCALIUC AND Z. GRUJIĆ
}

\begin{abstract}
Rigorous estimates for the total - (kinetic) energy plus pressure - flux in $\mathbb{R}^{3}$ are obtained from the three dimensional Navier-Stokes equations. The bounds are used to establish a condition - involving Taylor length scale and the size of the domain - sufficient for existence of the inertial range and the energy cascade in decaying turbulence (zero driving force, non-increasing global energy). Several manifestations of the locality of the flux under this condition are obtained. All the scales involved are actual physical scales in $\mathbb{R}^{3}$ and no regularity or homogeneity/scaling assumptions are made.
\end{abstract}

\section{INTRODUCTION}

One of the main features of Kolmogorov's empirical turbulence theory [11, 12, 10, is existence of energy cascade over a wide range of length scales, called the inertial range, where the dissipation effects are dominated by the transport of energy from higher to lower scales. Energy cascades have been observed in physical experiments, but theoretical justification of this phenomenon using equations of fluid motion, and in particular, the Navier-Stokes equations (NSE), remains far from being settled. The technical complexity of the NSE makes it difficult to establish conditions under which such cascades can occur. A particular problem is the possible lack of regularity of the solutions to the NSE, and thus choosing the right setting becomes crucial. (For an overview of various mathematical models of turbulence and the theory of the NSE, see, e.g., [7, 9, 6] and [14, 4, 18, respectively.)

The first studies in this direction were made in 8 , where infinite-time averages of the Leray-Hopf solutions in the Fourier setting were used to establish a sufficient condition for the energy cascade. This condition, involving Taylor length scale, provided an inspiration for the sufficient condition (4.13) obtained in section 4. In contrast to [8], our goal was to work in physical space, dealing with actual length scales in $\mathbb{R}^{3}$ rather than the Fourier wave numbers.

In studying a PDE model, a natural way of introducing a concept of scale is to measure oscillations, i.e., (distributional) derivatives of a quantity with respect to the scale.

Considering an $L_{\text {loc }}^{1}$ function $f$ on a ball of radius $2 R, B\left(\mathbf{x}_{0}, 2 R\right)$, the physical scale $R$ is introduced via bounds on the distributional derivatives of $f$ where a test function $\psi$ is a refined - smooth, non-negative, equal to 1 on $B\left(\mathbf{x}_{0}, R\right)$ and featuring optimal bounds on the derivatives over the outer $R$-layer - cut-off function on $B\left(\mathbf{x}_{0}, 2 R\right)$. (Uniformity in all scales dictates linearity of the length of the outer layer in $R$; hence $B\left(\mathbf{x}_{0}, R+R\right)$.)

Date: November 15, 2018. 
More explicitly,

$$
\left|\left(D^{\alpha} f, \psi\right)\right| \leq \int_{B\left(\mathbf{x}_{0}, 2 R\right)}|f|\left|D^{\alpha} \psi\right| \leq\left(c(\alpha) \frac{1}{R^{|\alpha|}}|f|, \psi^{\rho(\alpha)}\right)
$$

for some $c(\alpha)>0$ and $\rho(\alpha)$ in $(0,1)$.

(An attempt to introduce a concept of scale via characteristic functions in place of smooth cut-off functions would lead to infinite concentration - delta functions invalidating much of the desired calculus.)

This approach has a similar flavor as introducing the Fourier scale $|\xi|$ via

$$
\widehat{D^{\alpha} f}(\xi)=i^{|\alpha|} \xi^{\alpha} \hat{f}(\xi)
$$

(in the Schwarz space, and then by duality in the space of tempered distributions).

Let $\mathbf{x}_{0}$ be in $B\left(\mathbf{0}, R_{0}\right)\left(R_{0}\right.$ being the integral scale, $B\left(\mathbf{0}, 2 R_{0}\right) \subset \Omega$ where $\Omega$ is the global spatial domain) and $0<R \leq R_{0}$. Define local - per unit of mass - (kinetic) energy, e and enstrophy, $\mathrm{E}$, at time $t$, associated with the ball $B\left(\mathbf{x}_{0}, R\right)$ by

$$
\begin{aligned}
& \mathrm{e}_{\mathbf{x}_{0}, R}(t)=\int \frac{1}{2}|\mathbf{u}|^{2} \phi^{2 \delta-1} d \mathbf{x}, \\
& \mathrm{E}_{\mathbf{x}_{0}, R}(t)=\int|\nabla \otimes \mathbf{u}|^{2} \phi d \mathbf{x},
\end{aligned}
$$

where $\phi=\eta \psi$ and $\eta$ and $\psi$ are refined cut-off functions in time and space, respectively (for some $\frac{1}{2}<\delta<1$ ).

A total flux - (kinetic) energy plus pressure - through the boundary of a region $D$ is given by

$$
\int_{\partial D}\left(\frac{1}{2}|\mathbf{u}|^{2}+p\right) \mathbf{u} \cdot \mathbf{n} d s=\int_{D}[(\mathbf{u} \cdot \nabla) \mathbf{u}+\nabla p] \cdot \mathbf{u} d \mathbf{x}
$$

where $\mathbf{n}$ is an outward normal. Considering the NSE localized to $B\left(\mathbf{x}_{0}, 2 R\right)$ - and utilizing $\nabla \cdot \mathbf{u}=0$ - leads to a localized flux,

$$
\Phi_{\mathbf{x}_{0}, R}(t)=\int\left(\frac{1}{2}|\mathbf{u}|^{2}+p\right) \mathbf{u} \cdot \nabla \phi d \mathbf{x}=-\int[(\mathbf{u} \cdot \nabla) \mathbf{u}+\nabla p] \cdot \mathbf{u} \phi d \mathbf{x} .
$$

Since $\psi$ can be constructed such that $\nabla \phi=\eta \nabla \psi$ is oriented along the radial directions of $B\left(\mathbf{x}_{0}, 2 R\right)$ toward the center of the ball, $\Phi_{\mathbf{x}_{0}, R}$ represents the flux into $B\left(\mathbf{x}_{0}, R\right)$ through the layer between the spheres $S\left(\mathbf{x}_{0}, 2 R\right)$ and $S\left(\mathbf{x}_{0}, R\right)(\nabla \phi \equiv 0$ on $\left.B\left(\mathbf{x}_{0}, R\right)\right)$.

A more dynamic physical significance of the sign of $\Phi_{\mathbf{x}_{0}, R}$ can be seen from the equations: multiplying the NSE by $\psi \mathbf{u}$ and integrating over $B\left(\mathbf{x}_{0}, 2 R\right)$ (formally, assuming smoothness) leads to

$$
\frac{d}{d t} \int \frac{1}{2}|\mathbf{u}|^{2} \psi d \mathbf{x}=\Phi_{\mathbf{x}_{0}, R}+\nu \int \triangle \mathbf{u} \cdot \mathbf{u} \psi d \mathbf{x} .
$$

Plainly, the positivity of $\Phi_{\mathbf{x}_{0}, R}$ contributes to the increase of the kinetic energy around the point $\mathbf{x}_{0}$ at scale $R$.

Since the flux consists of both the kinetic and the pressure parts, a natural question is whether there is a transfer of the kinetic energy from larger scales into $B\left(\mathbf{x}_{0}, R\right)$, or perhaps the increase is mainly due to the change in pressure. In general, it is possible that the increase of the kinetic energy around $\mathbf{x}_{0}$ is due solely to 
the pressure part; a simple example being $\mathbf{u}=(c, c, c) t, p=c x_{1}+c x_{2}+c x_{3}$. However, in physical situations where the kinetic energy on the (global) spatial domain $\Omega$ is non-increasing, e.g., a bounded domain with no-slip boundary conditions, or the whole space with either decay at infinity or periodic boundary conditions (here, we are concerned with the case of decaying turbulence, setting the driving force to zero), the increase of the kinetic energy in $B\left(\mathbf{x}_{0}, R\right)$ - and consequently, the positivity of $\Phi_{\mathbf{x}_{0}, R}$ - implies local transfer of the kinetic energy from larger scales simply because the local kinetic energy is increasing while the global kinetic energy is nonincreasing resulting in decrease of the kinetic energy in the complement. This is also consistent with the fact that in the aforementioned scenarios one can project the NSE - in an appropriate functional space - to the subspace of divergence-free functions effectively eliminating the pressure and revealing that the local flux $\Phi_{\mathbf{x}_{0}, R}$ is indeed driven by transport/inertial effects rather than the change in the pressure. (The $\mathbf{u}=(c, c, c) t, p=c x_{1}+c x_{2}+c x_{3}$ example pertains to a completely opposite situation, the kinetic energy is simply uniformly growing over the whole spatial domain.)

Henceforth, following the discussion in the preceding paragraphs - in the setting of decaying turbulence (zero driving force, non-increasing global energy) - the positivity and the negativity of $\Phi_{\mathbf{x}_{0}, R}$ will be interpreted as a transfer of (kinetic) energy around the point $\mathbf{x}_{0}$ at scale $R$ toward smaller scales and a transfer of (kinetic) energy around the point $\mathbf{x}_{0}$ at scale $R$ toward larger scales, respectively.

Completely analogous definitions hold for shells of radii $R$ and $2 R$.

We also consider finite time averages of each of the aforementioned quantities.

Our goal is to obtain a manifestation of the (kinetic) energy cascade in physical space, i.e., formulate a condition on $B\left(\mathbf{0}, R_{0}\right)$ that would imply that the timeaveraged energy transfers/cascades to smaller scales across a range of scales (the existence of the inertial range).

A key point here is that we do not assume any homogeneity of the flow; hence one can not expect to show that the local fluxes are positive for each individual ball $B(\mathbf{x}, R)$. The best one can hope for is to prove the positivity of the flux over some spatial average.

We choose to work with a very straightforward spatial average: the arithmetic mean of the local fluxes - time-averaged, per unit mass - computed over a family of coverings of $B\left(\mathbf{0}, R_{0}\right)$, the so-called optimal coverings.

Let $K_{1}$ and $K_{2}$ be two positive integers. A covering $\left\{B\left(\mathbf{x}_{i}, R\right)\right\}_{i=1}^{n}$ of $B\left(\mathbf{0}, R_{0}\right)$ is an optimal covering (with parameters $K_{1}$ and $K_{2}$ ) if

$$
\left(\frac{R_{0}}{R}\right)^{3} \leq n \leq K_{1}\left(\frac{R_{0}}{R}\right)^{3},
$$

and any point $\mathbf{x}$ in $B\left(\mathbf{0}, R_{0}\right)$ is covered by at most $K_{2}$ balls $B\left(\mathbf{x}_{i}, 2 R\right)$. (Optimal coverings exist for all large enough $K_{1}$ and $K_{2}$, the critical values depending only on dimension of the space. In $\mathbb{R}^{3}$, we can take $K_{1}=K_{2}=8$.)

Let $f$ be a sign-varying quantity (e.g., the flux density $-[(\mathbf{u} \cdot \nabla) \mathbf{u}+\nabla p] \cdot \mathbf{u})$, and consider the arithmetic mean of the quantity locally averaged over the (optimal) covering elements $B\left(\mathbf{x}_{i}, R\right)$,

$$
F_{R}=\frac{1}{n} \sum_{i=1}^{n} \frac{1}{R^{3}} \int_{B\left(\mathbf{x}_{i}, 2 R\right)} f \psi_{i}^{\rho} d \mathbf{x}
$$


(for some $0<\rho \leq 1$ ).

A revealing observation is that $F_{R} \sim \operatorname{const}(R)$ for all optimal coverings at scale $R\left(K_{1}\right.$ and $K_{2}$ fixed) indicates there are no significant fluctuations of sign of $f$ at scales comparable or greater than $R$. In other words, if there are significant fluctuations of sign of $f$ at scale $R^{*}, F_{R}$ will run over a wide range of values while the average is being run over all permissible optimal coverings (determined by $K_{1}$ and $K_{2}$ ), for any $R$ comparable or less than $R^{*}$.

When there is no change of sign at all, i.e., in the case of a signed quantity (e.g., the energy density $f=\frac{1}{2}|\mathbf{u}|^{2}, \rho=2 \delta-1$ or the enstrophy density $f=|\nabla \otimes \mathbf{u}|^{2}, \rho=$ 1 ), one would then expect that for any scale $R, 0<R \leq R_{0}$, the averages $F_{R}$ are all comparable to each other. This is in fact true (an easy proof).

Utilizing the NSE via the local energy inequality - in the mathematical setting of suitable weak solutions [17, 1] - we establish the positivity and near-constancy (comparable to $\nu \mathrm{E}$, where $\nu$ is the viscosity and $\mathrm{E}$ is the average enstrophy over $\left.B\left(\mathbf{0}, 2 R_{0}\right) \times(0,2 T)\right)$ of the averaged flux across a range of scales under a very simple and natural (in the sense of turbulence phenomenology) condition; namely, that Taylor micro-scale $\tau_{0}$ associated with $B\left(\mathbf{0}, R_{0}\right)$ is smaller than the integral spatial scale $R_{0}$ (cf. (4.13) ). The larger the gap, the deeper the inertial range. This condition is reminiscent of the Poincaré inequality on a domain of the corresponding size (see Remark 4.2); moreover, the condition in hand would be easy to check in physical experiments as the averages involved are very straightforward. In addition, the length of the time interval $T$ is consistent with the intrinsic scaling of the model (cf. (4.2)).

It is interesting to interpret the cascade in the light of the above observation regarding the meaning of near-constancy of optimal cover averages. Essentially, for any $\tau_{0} \leq R \leq R_{0}$ (within the inertial range), the flux density does not experience significant fluctuations of sign at scale $R$; the significant fluctuations of sign are only possible at the scales substantially smaller than $\tau_{0}$, i.e., inside the dissipation range.

The second part of the paper concerns locality of the flux. It is believed (see [16]) that the energy flux inside the inertial range of turbulent flows depends strongly on the flow in nearby scales, its dependence on the lower and much higher scales being weak. The theoretical proof of this conjecture remained elusive. The first quantitative results on fluxes were obtained by early 70's (see [13]). Much later, the authors in [15] used the NSE in the Fourier setting to explore locality of scale interactions for statistical averages, while the investigation in [5] revealed the locality of filtered energy flux under an assumption that solutions to the vanishing viscosity Euler's equations saturate a defining inequality of a suitable Besov space, i.e., under a (weak) scaling assumption. A more recent work [2] provided a proof of the locality of the energy flux in the setting of the Littlewood-Paley decomposition.

In the last section we prove the locality of the energy cascade - in decaying turbulence - in the physical space throughout the inertial range established in Theorem 4.1. In particular, considering dyadic shells at the scales $2^{k} R$ ( $k$ an integer) in the physical space, we show that both ultraviolet and infrared locality propagate exponentially in the shell number $k$.

To the best of our knowledge, the condition (4.13) is presently the only condition (in any solution setting) implying both the existence of the inertial range and the 
locality of the energy flux. Moreover, it does not involve any additional regularity or homogeneity/scaling assumptions on the solutions to the NSE.

\section{PRELIMINARIES}

We consider three dimensional incompressible Navier-Stokes equations (NSE)

$$
\begin{aligned}
\frac{\partial}{\partial t} \mathbf{u}(t, \mathbf{x})-\nu \Delta \mathbf{u}(t, \mathbf{x})+(\mathbf{u}(t, \mathbf{x}) \cdot \nabla) \mathbf{u}(t, \mathbf{x})+\nabla p(t, \mathbf{x}) & =0 \\
\nabla \cdot \mathbf{u}(t, \mathbf{x}) & =0
\end{aligned}
$$

where the space variable $\mathbf{x}$ is in $\mathbb{R}^{3}$ and the time variable $t$ is in $(0, \infty)$. The vectorvalued function $\mathbf{u}$ and the scalar-valued function $p$ represent the fluid velocity and the pressure, respectively, while the constant $\nu$ is the viscosity of the fluid.

Since our goal is to investigate local fluxes in the physical space, the class of suitable weak solutions (see 1, 14]) will provide an appropriate mathematical framework.

Definition 2.1. Let $\Omega$ be an open connected set in $\mathbb{R}^{3}$. We say that $(\mathbf{u}, p)$ is a suitable weak solution on $(0, \infty) \times \Omega$ if

(a) $\mathbf{u} \in L^{\infty}\left((0, \infty), L^{2}(\Omega)^{3}\right) \cap L^{2}\left((0, \infty), H^{1}(\Omega)^{3}\right)$ and $p \in L^{3 / 2}((0, \infty) \times \Omega)$;

(b) the NSE (2.1) are satisfied in the weak (distributional) sense;

(c) the local energy inequality is satisfied: for any $\phi \in \mathcal{D}((0, \infty) \times \Omega), \phi \geq 0$ we have

$$
2 \nu \iint|\nabla \otimes \mathbf{u}|^{2} \phi d \mathbf{x} d t \leq \iint|\mathbf{u}|^{2}\left(\partial_{t} \phi+\nu \Delta \phi\right) d \mathbf{x} d t+\iint\left(|\mathbf{u}|^{2}+2 p\right) \mathbf{u} \cdot \nabla \phi d \mathbf{x} d t
$$

where $\mathcal{D}((0, \infty) \times \Omega)$ denotes the space of infinitely differentiable functions with compact support in $(0, \infty) \times \Omega$.

The existence of the suitable weak solutions in the case where $\Omega=\mathbb{R}^{3}$ and the external force is zero, given a divergence-free initial condition in $L^{2}$, was first established in [17. See also 1, 14] for more general results related to existence and regularity properties of the suitable weak solutions.

A solution to the NSE on $(0, \infty) \times \Omega$ is called regular if its $H^{1}$ norm is bounded on $(0, T)$ for any $T$ positive. Given appropriate boundary conditions, this implies that the solution is infinitely differentiable (in fact, analytic) in both space and time and so it is a classical physical solution. In particular, the local energy equality holds (2.2) becomes an equality). The smoothness of the suitable weak solutions to the NSE is still an open problem, and the best result in this direction reads that the one-dimensional (parabolic) Hausdorff measure of the singular set in $(0, T) \times \Omega$ is zero [1] (outside the singular set, a suitable weak solution is infinitely differentiable in the spatial variables).

In what follows, we consider

$$
R_{0}>0 \text { such that } B\left(\mathbf{0}, 3 R_{0}\right) \subset \Omega
$$

where $B\left(\mathbf{0}, 3 R_{0}\right)$ denotes the ball in $\mathbb{R}^{3}$ centered at the origin and with the radius $3 R_{0}$.

Let $1 / 2 \leq \delta<1$. Choose $\psi_{0} \in \mathcal{D}\left(B\left(\mathbf{0}, 2 R_{0}\right)\right)$ satisfying

$$
0 \leq \psi_{0} \leq 1, \quad \psi_{0}=1 \text { on } B\left(\mathbf{0}, R_{0}\right), \quad \frac{\left|\nabla \psi_{0}\right|}{\psi_{0}^{\delta}} \leq \frac{C_{0}}{R_{0}}, \quad \frac{\left|\triangle \psi_{0}\right|}{\psi_{0}^{2 \delta-1}} \leq \frac{C_{0}}{R_{0}^{2}} .
$$




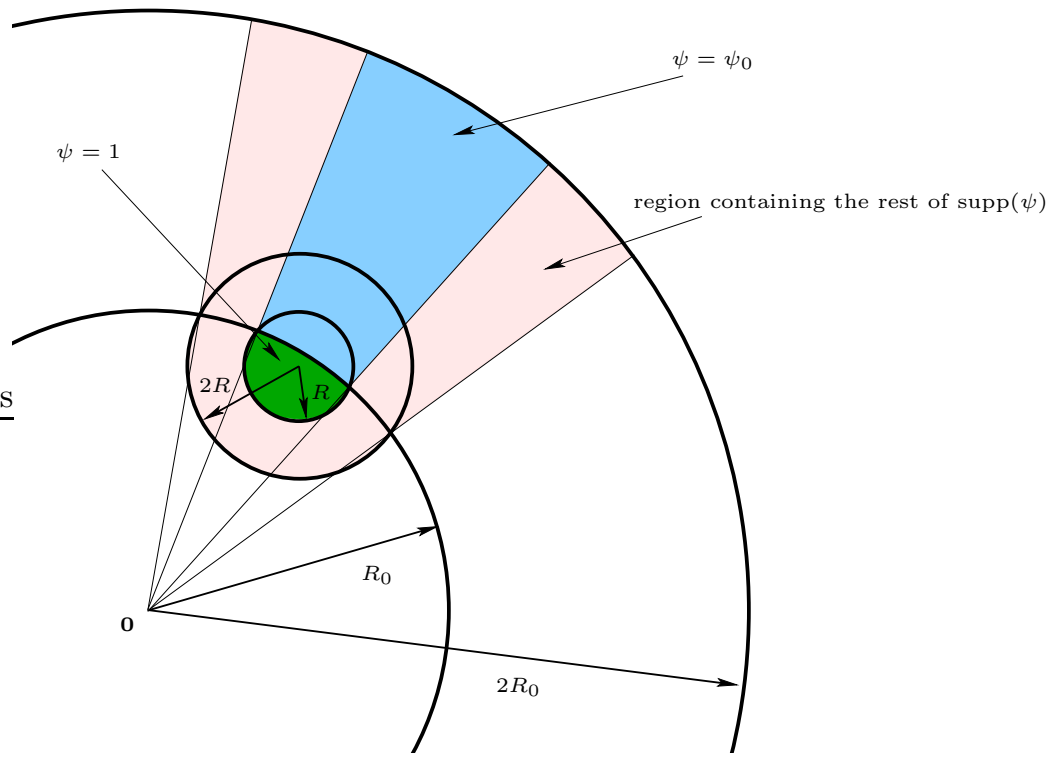

Figure 1. Regions of $\operatorname{supp}(\psi)$ in the case $B\left(\mathbf{x}_{0}, R\right) \not \subset B\left(\mathbf{0}, R_{0}\right)$, cross-section.

For a $T>0$ (to be chosen later), $\mathbf{x}_{0} \in B\left(\mathbf{0}, R_{0}\right)$ and $0<R \leq R_{0}$ define $\phi=\phi_{\mathbf{x}_{0}, T, R}(t, \mathbf{x})=\eta(t) \psi(\mathbf{x})$ to be used in the local energy inequality (2.2) where $\eta=\eta_{T}(t)$ and $\psi=\psi_{\mathbf{x}_{0}, R}(\mathbf{x})$ are refined cut-off functions satisfying the following conditions,

$$
\eta \in \mathcal{D}(0,2 T), \quad 0 \leq \eta \leq 1, \quad \eta=1 \text { on }(T / 4,5 T / 4), \quad \frac{\left|\eta^{\prime}\right|}{\eta^{\delta}} \leq \frac{C_{0}}{T} ;
$$

if $B\left(\mathbf{x}_{0}, R\right) \subset B\left(\mathbf{0}, R_{0}\right)$, then $\psi \in \mathcal{D}\left(B\left(\mathbf{x}_{0}, 2 R\right)\right)$ with

$$
0 \leq \psi \leq \psi_{0}, \quad \psi=1 \text { on } B\left(\mathbf{x}_{0}, R\right) \cap B\left(\mathbf{0}, R_{0}\right), \quad \frac{|\nabla \psi|}{\psi^{\delta}} \leq \frac{C_{0}}{R}, \frac{|\triangle \psi|}{\psi^{2 \delta-1}} \leq \frac{C_{0}}{R^{2}},
$$

and if $B\left(\mathbf{x}_{0}, R\right) \not \subset B\left(\mathbf{0}, R_{0}\right)$, then $\psi \in \mathcal{D}\left(B\left(\mathbf{0}, 2 R_{0}\right)\right)$ with $\psi=1$ on $B\left(\mathbf{x}_{0}, R\right) \cap$ $B\left(\mathbf{0}, R_{0}\right)$ satisfying, in addition to (2.6), the following:

$$
\begin{aligned}
& \psi=\psi_{0} \text { on the part of the cone in } \mathbb{R}^{3} \text { centered at zero and passing through } \\
& S\left(\mathbf{0}, R_{0}\right) \cap B\left(\mathbf{x}_{0}, R\right) \text { between } S\left(\mathbf{0}, R_{0}\right) \text { and } S\left(\mathbf{0}, 2 R_{0}\right)
\end{aligned}
$$

and

$$
\psi=0 \text { on } B\left(\mathbf{0}, R_{0}\right) \backslash B\left(\mathbf{x}_{0}, 2 R\right) \text { and outside the part of the cone in } \mathbb{R}^{3}
$$

$$
\text { centered at zero and passing through } S\left(\mathbf{0}, R_{0}\right) \cap B\left(\mathbf{x}_{0}, 2 R\right)
$$

between $S\left(\mathbf{0}, R_{0}\right)$ and $S\left(\mathbf{0}, 2 R_{0}\right)$.

Figure 1 illustrates the definition of $\psi$ in the case $B\left(\mathbf{x}_{0}, R\right)$ is not entirely contained in $B\left(\mathbf{0}, R_{0}\right)$.

Remark 2.1. The additional conditions on the boundary elements (2.7) and (2.8) are necessary to obtain the lower bound on the fluxes in terms of the same version of the localized enstrophy $E$ in Theorems 4.1 and 5.2 (see Remarks 4.4 and 5.5). 


\section{Localized Energy, Enstrophy and Flux; Ensemble Averages}

Let $\mathbf{x}_{0} \in B\left(\mathbf{0}, R_{0}\right)$ and $0<R \leq R_{0}$. Define localized energy, e, and enstrophy, $\mathrm{E}$, at time $t$ - all per unit of mass - associated with $B\left(\mathbf{x}_{0}, R\right)$ by

$$
\begin{gathered}
\mathrm{e}_{\mathbf{x}_{0}, R}(t)=\int \frac{1}{2}|\mathbf{u}|^{2} \phi^{2 \delta-1} d \mathbf{x}, \\
\mathrm{E}_{\mathbf{x}_{0}, R}(t)=\int|\nabla \otimes \mathbf{u}|^{2} \phi d \mathbf{x}
\end{gathered}
$$

(for some $\frac{1}{2}<\delta<1$ ). by

The total - (kinetic) energy plus pressure - flux through sphere $S\left(\mathbf{x}_{0}, R\right)$ is given

$$
\int_{S\left(\mathbf{x}_{0}, R\right)}\left(\frac{1}{2}|\mathbf{u}|^{2}+p\right) \mathbf{u} \cdot \mathbf{n} d s=\int_{B\left(\mathbf{x}_{0}, R\right)}[(\mathbf{u} \cdot \nabla) \mathbf{u}+\nabla p] \cdot \mathbf{u} d \mathbf{x}
$$

where $\mathbf{n}$ is an outward normal. Considering the NSE localized to $B\left(\mathbf{x}_{0}, R\right)$ leads to a localized version of the flux,

$$
\Phi_{\mathbf{x}_{0}, R}(t)=\int\left(\frac{1}{2}|\mathbf{u}|^{2}+p\right) \mathbf{u} \cdot \nabla \phi d \mathbf{x}=-\int[(\mathbf{u} \cdot \nabla) \mathbf{u}+\nabla p] \cdot \mathbf{u} \phi d \mathbf{x}
$$

where $\phi=\eta \psi$ with $\eta$ and $\psi$ as in (2.5) 2.6). Since $\psi$ can be constructed such that $\nabla \phi=\eta \nabla \psi$ is oriented along the radial directions of $B\left(\mathbf{x}_{0}, R\right)$ towards the center of the ball $\mathbf{x}_{0}, \Phi\left(\mathbf{x}_{0}, R\right)$ represents the flux into $B\left(\mathbf{x}_{0}, R\right)$ through the layer between the spheres $S\left(\mathbf{x}_{0}, 2 R\right)$ and $S\left(\mathbf{x}_{0}, R\right)$ (in the case of the boundary elements satisfying the additional hypotheses (2.7) and (2.8), $\psi$ is almost radial and the gradient still points inward).

For a quantity $\Theta_{\mathbf{x}, R}(t), t \in[0,2 T]$ and a covering $\left\{B\left(\mathbf{x}_{i}, R\right)\right\}_{i=1, n}$ of $B\left(\mathbf{0}, R_{0}\right)$ define a time-space ensemble average

$$
\langle\Theta\rangle_{R}=\frac{1}{T} \int \frac{1}{n} \sum_{i=1}^{n} \frac{1}{R^{3}} \Theta_{\mathbf{x}_{i}, R}(t) d t .
$$

Denote by

$$
\begin{aligned}
\mathrm{e}_{R} & =\left\langle\mathrm{e}_{\mathbf{x}, R}(t)\right\rangle_{R}, \\
\mathrm{E}_{R} & =\left\langle\mathrm{E}_{\mathbf{x}, R}(t)\right\rangle_{R}, \\
\Phi_{R} & =\left\langle\Phi_{\mathbf{x}, R}(t)\right\rangle_{R},
\end{aligned}
$$

the averaged localized energy, enstrophy and inward-directed flux over balls of radius $R$ covering $B\left(\mathbf{0}, R_{0}\right)$.

Also, introduce the time-space average of the localized energy on $B\left(\mathbf{0}, R_{0}\right)$,

$$
\mathrm{e}=\frac{1}{T} \int \frac{1}{R_{0}^{3}} \mathrm{e}_{\mathbf{0}, R_{0}}(t) d t=\frac{1}{T} \frac{1}{R_{0}^{3}} \iint \frac{1}{2}|\mathbf{u}|^{2} \phi_{0}^{2 \delta-1} d \mathbf{x} d t
$$

and the time-space average of the localized enstrophy on $B\left(\mathbf{0}, R_{0}\right)$,

$$
\mathrm{E}=\frac{1}{T} \int \frac{1}{R_{0}^{3}} \mathrm{E}_{\mathbf{0}, R_{0}}(t) d t=\frac{1}{T} \frac{1}{R_{0}^{3}} \iint|\nabla \otimes \mathbf{u}|^{2} \phi_{0} d \mathbf{x} d t
$$

where

$$
\phi_{0}(t, \mathbf{x})=\eta(t) \psi_{0}(\mathbf{x})
$$


with $\psi_{0}$ defined in (2.4).

Finally, define Taylor length scale associated with $B\left(\mathbf{0}, R_{0}\right)$ by

$$
\tau_{0}=\left(\frac{\mathrm{e}}{\mathrm{E}}\right)^{1 / 2} .
$$

Note that the possible lack of regularity may produce additional loss of energy, resulting in anomalous energy dissipation and the loss of flux leading to the strict inequality in (2.2). Let us mention here that in the turbulence literature the term 'anomalous dissipation' is usually utilized in the context of the possible energy dissipation due to the (possible) singularities in the 3D Euler equations (the observation originally made by Onsager); for rigorous results on Onsager's conjecture on the energy conservation in the Euler equations see, e.g., [3], and a recent work [2].

Denote by $\Phi_{\mathbf{x}_{0}, R}^{\infty}$ the loss of flux due to possible singularities in $[0,2 T] \times B\left(\mathbf{x}_{0}, 2 R\right)$,

$$
\begin{aligned}
& \iint\left(\frac{1}{2}|\mathbf{u}|^{2}+p\right) \mathbf{u} \cdot \nabla \phi d \mathbf{x} d t-\Phi_{\mathbf{x}_{0}, R}^{\infty} \\
& =\nu \iint|\nabla \otimes \mathbf{u}|^{2} \phi d \mathbf{x} d t-\frac{1}{2} \iint|\mathbf{u}|^{2}\left(\partial_{t} \phi+\nu \Delta \phi\right) d \mathbf{x} d t
\end{aligned}
$$

where $\phi=\eta \psi$ with $\eta$ and $\psi$ as in (2.5) and (2.6 2.8). In particular, denote by $\Phi_{\infty}=\Phi_{\mathbf{0} R_{0}}^{\infty}$ the loss of flux due to singularities in $[0,2 T] \times B\left(\mathbf{0}, 2 R_{0}\right)$.

We will also consider the time-space ensemble averages of these anomalous fluxes,

$$
\Phi_{R}^{\infty}=\frac{1}{n} \sum_{i=1}^{n} \frac{1}{T} \frac{1}{R^{3}} \Phi_{\mathbf{x}_{i}, R}^{\infty} .
$$

Note that due to (2.2), all the anomalous fluxes are nonnegative,

$$
\Phi_{\mathbf{x}_{0}, R}^{\infty} \geq 0, \quad \Phi_{\infty} \geq 0, \quad \Phi_{R}^{\infty} \geq 0 ;
$$

they are all zero provided the equality holds in (2.2) inside $[0,2 T] \times B\left(\mathbf{0}, 2 R_{0}\right)$. In particular, the anomalous fluxes are all zero provided the solution in view is regular on $[0,2 T] \times B\left(\mathbf{0}, 2 R_{0}\right)$.

Consequently, the total localized flux into $B\left(\mathbf{x}_{0}, R\right)$ over interval $[0,2 T]$, including the (loss of) flux due to the possible loss of regularity, is

$$
\Psi_{\mathbf{x}_{0}, R}=\int \Phi_{\mathbf{x}_{0}, R}(t) d t-\Phi_{\mathbf{x}_{0}, R}^{\infty}
$$

and the time-space ensemble average of this flux at scales $R$ over time $T$ is

$$
\Psi_{R}=\Phi_{R}-\Phi_{R}^{\infty} .
$$

We will refer to $\Psi_{\mathbf{x}_{0}, R}$ and $\Psi_{R}$ as the modified flux over $[0,2 T]$ into $B\left(\mathbf{x}_{0}, R\right)$ and the (time-space ensemble) averaged modified flux at the scale $R$, respectively.

Let $K_{1}, K_{2}>1$ be two positive integers (independent of $R, R_{0}$, and any of the parameters of the NSE).

Definition 3.1. We say that a covering of $B\left(\mathbf{0}, R_{0}\right)$ by $n$ balls of radius $R$ is optimal if

$$
\left(\frac{R_{0}}{R}\right)^{3} \leq n \leq K_{1}\left(\frac{R_{0}}{R}\right)^{3} ;
$$

any $\mathbf{x} \in B\left(\mathbf{0}, R_{0}\right)$ is covered by at most $K_{2}$ balls $B\left(\mathbf{x}_{i}, 2 R\right)$. 
Note that optimal coverings exist for any $0<R \leq R_{0}$ provided $K_{1}$ and $K_{2}$ are large enough. In fact, the choice of $K_{1}$ and $K_{2}$ depends only on dimension of the space; in $\mathbb{R}^{3}$ we can choose $K_{1}=K_{2}=8$.

Henceforth, we assume that the averages $\langle\cdot\rangle_{R}$ are taken with respect to optimal coverings.

Lemma 3.1. If the covering $\left\{B\left(\mathbf{x}_{i}, R\right)\right\}_{i=1, n}$ of $B\left(\mathbf{0}, R_{0}\right)$ is optimal then

$$
\Phi_{R}^{\infty} \leq K \frac{1}{T} \frac{1}{R_{0}^{3}} \Phi_{\infty}
$$

where $K>0$ is a constant depending only on $K_{2}$ and dimension of the space $\mathbb{R}^{3}$.

Proof. Let $\left\{\mathbf{x}_{i_{j}}\right\}$ be a subset of $\left\{\mathbf{x}_{i}\right\}_{i=1, n}$ such that interiors of the balls $B\left(\mathbf{x}_{i_{j}}, 2 R\right)$ are pairwise disjoint. Using (3.12), we obtain

$$
\begin{aligned}
& \iint\left(\frac{1}{2}|\mathbf{u}|^{2}+p\right) \mathbf{u} \cdot \nabla \phi_{0} d \mathbf{x} d t-\Phi_{\infty} \\
& =\nu \iint|\nabla \otimes \mathbf{u}|^{2} \phi_{0} d \mathbf{x} d t-\frac{1}{2} \iint|\mathbf{u}|^{2}\left(\partial_{t} \phi_{0}+\nu \Delta \phi_{0}\right) d \mathbf{x} d t
\end{aligned}
$$

and

$$
\begin{aligned}
& \iint\left(\frac{1}{2}|\mathbf{u}|^{2}+p\right) \mathbf{u} \cdot \nabla\left(\sum_{j} \phi_{i_{j}}\right) d \mathbf{x} d t-\sum_{j} \Phi_{\mathbf{x}_{i_{j}}, R}^{\infty} \\
& =\nu \iint|\nabla \otimes \mathbf{u}|^{2}\left(\sum_{j} \phi_{i_{j}}\right) d \mathbf{x} d t-\frac{1}{2} \iint|\mathbf{u}|^{2}\left[\partial_{t}\left(\sum_{j} \phi_{i_{j}}\right)+\nu \Delta\left(\sum_{j} \phi_{i_{j}}\right)\right] d \mathbf{x} d t
\end{aligned}
$$

where $\phi_{0}=\eta \psi_{0}$ and $\phi_{i_{j}}=\eta \psi_{i_{j}}$ with $\eta$ as in (2.5), $\psi_{0}$ as in (2.4) and $\psi_{i_{j}}$ a test function corresponding to $B\left(\mathbf{x}_{i_{j}}, R\right)$ satisfying (2.6]2.8).

Note that the definitions of $\phi_{0}$ and $\phi_{i_{j}}$ imply

$$
\tilde{\phi}=\phi_{0}-\sum_{j} \phi_{i_{j}} \geq 0
$$

hence, by the local energy inequality (2.2),

$$
\begin{aligned}
& \iint\left(\frac{1}{2}|\mathbf{u}|^{2}+p\right) \mathbf{u} \cdot \nabla \tilde{\phi} d \mathbf{x} d t \\
& \geq \nu \iint|\nabla \otimes \mathbf{u}|^{2} \tilde{\phi} d \mathbf{x} d t-\frac{1}{2} \iint|\mathbf{u}|^{2}\left(\partial_{t} \tilde{\phi}+\nu \Delta \tilde{\phi}\right) d \mathbf{x} d t .
\end{aligned}
$$

If we add relations (3.21) and (3.22) and then subtract (3.20) we obtain

$$
\Phi_{\infty} \geq \sum_{j} \Phi_{\mathbf{x}_{i_{j}}, R}^{\infty}
$$

Let $\mathcal{L}$ be a cubic lattice inside $B\left(\mathbf{0}, R_{0}\right)$ with the points situated at the vertices of cubes of side $R / 2$ (Note that this lattice can be chosen such that the number of points in it is between $2^{3}\left(R_{0} / R\right)^{3}$ and $\left.(4 \pi / 3) 2^{3}\left(R_{0} / R\right)^{3}\right)$.

Since the covering $\left\{B\left(\mathbf{x}_{i}, R\right)\right\}$ is optimal, each point in $\mathcal{L}$ is contained in at most $K_{2}$ balls. Moreover, any ball in the covering will contain at least one point from the lattice.

If $\mathcal{L}^{\prime}$ is sub-lattice of $\mathcal{L}$ with points at vertices of cubes of side $4 R$, then the interiors of balls of radius $2 R$ containing different points of $\mathcal{L}^{\prime}$ are pairwise disjoint, 
and thus if we denote by $B\left(\mathbf{x}_{i_{p}}, R\right)$ a ball from the covering $\left\{B\left(\mathbf{x}_{i}, R\right)\right\}$ containing the point $p \in \mathcal{L}^{\prime}$, by (3.22),

$$
\Phi_{\infty} \geq \sum_{p \in \mathcal{L}^{\prime}} \Phi_{\mathbf{x}_{i_{p}}, R}^{\infty}
$$

Note that for each point $p \in \mathcal{L}^{\prime}$ there are at most $K_{2}$ choices for $B\left(\mathbf{x}_{i_{p}}, R\right)$. So

$$
K_{2} \Phi_{\infty} \geq \sum_{i: B\left(\mathbf{x}_{i}, R\right) \cap \mathcal{L}^{\prime} \neq \emptyset} \Phi_{\mathbf{x}_{i}, R}^{\infty} .
$$

Clearly $\mathcal{L}$ can be written as a union of $8^{3}=256$ sub-lattices $\mathcal{L}_{k}^{\prime}, k=1, \ldots, 256$, each $\mathcal{L}_{k}^{\prime}$ having the same properties as $\mathcal{L}^{\prime}$. Thus,

$$
8^{3} K_{2} \Phi_{\infty} \geq \sum_{i=1}^{n} \Phi_{\mathbf{x}_{i}, R}^{\infty} .
$$

Consequently,

$$
\Phi_{R}^{\infty}=\frac{1}{T} \frac{1}{R^{3}} \frac{1}{n} \sum_{i=1}^{n} \Phi_{\mathbf{x}_{i}, R}^{\infty} \leq 8^{3} K_{2} \frac{1}{T} \frac{1}{R^{3}} \frac{1}{n} \Phi_{\infty} \leq 8^{3} K_{2} \frac{1}{T} \frac{1}{R_{0}^{3}} \Phi_{\infty}
$$

where the last inequality is due to $n$ satisfying (3.18).

According to the lemma, the time-space ensemble averages $\Phi_{R}^{\infty}$ taken over the optimal coverings at the scale $R$ are bounded, independently of $R$, by the average loss of flux due to possible singularities inside $B\left(\mathbf{0}, 2 R_{0}\right)$.

\section{ENERGy CASCADE}

Let $\left\{B\left(\mathbf{x}_{i}, R\right)\right\}_{i=1, n}$ be an optimal covering of $B\left(\mathbf{0}, R_{0}\right)$.

Note that the local energy equality (3.12) and the definitions of $\mathrm{E}_{R}, \Phi_{R}$ and $\Phi_{R}^{\infty}$ ( (3.6), (3.7) and (3.13) ) imply

$$
\Psi_{R}=\Phi_{R}-\Phi_{R}^{\infty}=\nu \mathrm{E}_{R}-\frac{1}{n} \sum_{i=1}^{n} \frac{1}{T} \frac{1}{R^{3}} \iint \frac{1}{2}|\mathbf{u}|^{2}\left(\partial_{t} \phi_{i}+\nu \Delta \phi_{i}\right) d \mathbf{x} d t
$$

where $\phi_{i}=\eta \psi_{i}$ and $\psi_{i}$ is the spatial cut-off on $B\left(\mathbf{x}_{i}, 2 R\right)$ satisfying (2.5) 2.8).

If

$$
T \geq \frac{R_{0}^{2}}{\nu}
$$

then for any $0<R \leq R_{0}$,

hence,

$$
\begin{gathered}
\left|\left(\phi_{i}\right)_{t}\right|=\left|\eta_{t} \psi_{i}\right| \leq C_{0} \frac{1}{T} \eta^{\delta} \psi_{i} \leq \nu \frac{C_{0}}{R^{2}} \phi_{i}^{2 \delta-1}, \\
\nu\left|\Delta \phi_{i}\right|=\nu\left|\eta \Delta \psi_{i}\right| \leq C_{0} \frac{\nu}{R^{2}} \eta \psi_{i}^{2 \delta-1} \leq \nu \frac{C_{0}}{R^{2}} \phi_{i}^{2 \delta-1}
\end{gathered}
$$

$$
\Psi_{R} \geq \nu \mathrm{E}_{R}-\nu \frac{C_{0}}{R^{2}} \mathrm{e}_{R}
$$

The optimality conditions (3.17) and (3.18) paired with (2.6 2.8) imply

$$
\mathrm{E}_{R} \geq \frac{1}{K_{1}} \mathrm{E}
$$


and

$$
\mathrm{e}_{R} \leq K_{2} \mathrm{e}
$$

Consequently,

$$
\Psi_{R} \geq \nu \frac{1}{K_{1}} \mathrm{E}-\nu \frac{C_{0} K_{2}}{R^{2}} \mathrm{e}
$$

leading to the following proposition.

\section{Proposition 4.1.}

$$
\Psi_{R} \geq c_{1} \nu \mathrm{E}\left(1-c_{2} \frac{\tau_{0}^{2}}{R^{2}}\right)
$$

with $c_{1}=1 / K_{1}$ and $c_{2}=C_{0} K_{1} K_{2}$ (provided conditions [3.17]3.18) are satisfied).

Suppose that

$$
\tau_{0}<\frac{\gamma}{c_{2}^{1 / 2}} R_{0}
$$

for some $0<\gamma<1$. Then, for any $R,\left(c_{2}^{1 / 2} / \gamma\right) \tau_{0} \leq R \leq R_{0}$,

$$
\Psi_{R} \geq c_{1}\left(1-\gamma^{2}\right) \nu \mathrm{E}=c_{0, \gamma} \nu \mathrm{E}
$$

where

$$
c_{0, \gamma}=c_{1}\left(1-\gamma^{2}\right)=\frac{1-\gamma^{2}}{K_{1}} .
$$

To obtain an upper bound on the averaged modified flux, note that for optimal coverings, in addition to (4.4),

$$
\mathrm{E}_{R} \leq K_{2} \mathrm{E} .
$$

Hence, (4.1) implies

$$
\Psi_{R} \leq \nu \mathrm{E}_{R}+\nu \frac{C_{0}}{R^{2}} \mathrm{e}_{R} \leq \nu K_{2} \mathrm{E}+\nu C_{0} K_{2} \frac{1}{R^{2}} \text { e. }
$$

If the condition (4.7) holds for some $0<\gamma<1$, then it follows that for any $R$, $\left(c_{2}{ }^{1 / 2} / \gamma\right) \tau_{0} \leq R \leq R_{0}$,

$$
\Psi_{R} \leq \nu K_{2} \mathrm{E}+\nu \frac{C_{0} K_{2} \gamma^{2}}{c_{2}} \mathrm{E} \leq c_{1, \gamma} \nu \mathrm{E}
$$

where

$$
c_{1, \gamma}=K_{2}\left[1+\frac{C_{0} \gamma^{2}}{c_{2}}\right]=K_{2}\left[1+\frac{\gamma^{2}}{K_{1} K_{2}}\right] .
$$

Thus we have proved the following.

Theorem 4.1. Assume that for some $0<\gamma<1$

$$
\tau_{0}<c \gamma R_{0},
$$

where

$$
c=\frac{1}{\sqrt{C_{0} K_{1} K_{2}}} .
$$


Then, for all $R$,

$$
\frac{1}{c \gamma} \tau_{0} \leq R \leq R_{0}
$$

the averaged modified flux $\Psi_{R}$ satisfies

$$
c_{0, \gamma} \nu \mathrm{E} \leq \Psi_{R} \leq c_{1, \gamma} \nu \mathrm{E}
$$

where

$$
c_{0, \gamma}=\frac{1-\gamma^{2}}{K_{1}}, \quad c_{1, \gamma}=K_{2}\left[1+\frac{\gamma^{2}}{K_{1} K_{2}}\right],
$$

and the average $\langle\cdot\rangle_{R}$ is computed over a time interval $[0,2 T]$ with $T \geq R_{0}^{2} / \nu$ and determined by an optimal covering of $B\left(\mathbf{0}, R_{0}\right)$ (i.e., a covering satisfying (3.17) and (3.18)).

Remark 4.1. As noted in the introduction - in the case the global energy is nonincreasing - the theorem provides a sufficient condition for the energy cascade; i.e., a nearly constant nonlinear transfer of time-averaged (kinetic) energy to smaller scales across the inertial range defined by (4.15).

More precisely, since we are working with weak solutions, the expression for the rate of change of local kinetic energy (1.1) morphs into

$$
-\iint \frac{1}{2}|\mathbf{u}|^{2} \phi_{t}=\Psi_{\mathbf{x}_{0}, R}+\text { viscous terms. }
$$

The interpretation remains the same, the only differences being that the timederivative of the local kinetic energy is taken in the sense of distributions and the flux got replaced with the modified flux to account for possible singularities.

Note that the averages are taken over finite-time intervals $[0,2 T]$ with $T \geq R_{0}^{2} / \nu$ (see (4.2) ). This lower bound on the length of the time interval $T$ is consistent with the picture of decaying turbulence; namely, small $\nu$ corresponds to the welldeveloped turbulence which then persists for a longer time and it makes sense to average over longer time-intervals.

Remark 4.2. In the language of turbulence, the condition (4.13) simply reads that the Taylor micro-scale computed over the domain in view is smaller than the integral scale (diameter of the domain).

On the other hand, (4.13) is equivalent to

$$
\frac{1}{T} \iint|\mathbf{u}|^{2} \phi_{0}^{2 \delta-1} d \mathbf{x} d t<\frac{\gamma^{2}}{C_{0} K_{1} K_{2}} R_{0}^{2} \frac{1}{T} \iint|\nabla \otimes \mathbf{u}|^{2} \phi_{0} d \mathbf{x} d t
$$

which can be read as a requirement that the time average of a Poincaré-like inequality on $B\left(\mathbf{0}, 2 R_{0}\right)$ is not saturating; this will hold for a variety of flows in the regions of active fluid (large gradients).

Remark 4.3. Since $\Phi_{R}^{\infty} \geq 0$, all the lower bounds on the averaged modified fluxes hold for the usual averaged fluxes $\Phi_{R}$; in particular,

$$
\Phi_{R} \geq c_{1} \nu \mathrm{E}\left(1-c_{2} \frac{\tau_{0}^{2}}{R^{2}}\right)
$$

and consequently, provided (4.13) and (4.15) hold,

$$
\Phi_{R} \geq c_{0, \gamma} \nu \mathrm{E} .
$$


Also, if a solution $\mathbf{u}$ is such that the (2.2) holds with equality and in particular, if the solution is regular, then all the estimates, including (4.16), hold for the usual averaged flux $\Phi_{R}$.

Remark 4.4. If we do not impose the additional assumptions (2.7) and (2.8) for the test functions on the balls $B\left(\mathbf{x}_{i}, R\right) \not \subset B\left(\mathbf{0}, R_{0}\right)$, then the lower bounds for $\Psi_{R}$ in (4.6) and (4.16) will hold with E replaced by the time-space average of the non-localized in space enstrophy on $B\left(\mathbf{0}, R_{0}\right)$,

$$
E^{\prime}=\frac{1}{T} \int_{0}^{2 T} \frac{1}{R_{0}^{3}} \int_{B\left(\mathbf{0}, R_{0}\right)}|\nabla \otimes \mathbf{u}|^{2} \eta d \mathbf{x} d t .
$$

This is the case because the estimate (4.3) gets replaced with

$$
\mathrm{E}_{R} \geq \frac{1}{K_{1}} E^{\prime}
$$

\section{Locality of the AVERAGed FluX}

Let $\mathbf{x}_{0} \in B\left(\mathbf{0}, R_{0}\right), 0<R_{2}<R_{1} \leq R_{0}$. In order to study the flux through the shell $A\left(\mathrm{x}_{0}, R_{1}, R_{2}\right)$ between the spheres $S\left(\mathrm{x}_{0}, R_{2}\right)$ and $S\left(\mathbf{x}_{0}, R_{1}\right)$, in what follows, we will consider the modified test functions $\phi=\phi_{\mathbf{x}_{0}, T, R_{1}, R_{2}}(t, \mathbf{x})=\eta(t) \psi(\mathbf{x})$ to be used in the local energy inequality (2.2) where $\eta=\eta_{T}(t)$ as in (2.5) and $\psi \in$ $\mathcal{D}\left(A\left(\mathbf{x}_{0}, 2 R_{1}, R_{2} / 2\right)\right)$ satisfying

$$
0 \leq \psi \leq \psi_{0}, \quad \psi=1 \text { on } A\left(\mathbf{x}_{0}, R_{1}, R_{2}\right), \quad \frac{|\nabla \psi|}{\psi^{\delta}} \leq \frac{C_{0}}{\tilde{R}}, \frac{|\triangle \psi|}{\psi^{2 \delta-1}} \leq \frac{C_{0}}{\tilde{R}^{2}}
$$

where $\psi_{0}$ is defined in (2.4) and

$$
\tilde{R}=\tilde{R}\left(R_{1}, R_{2}\right)=\min \left\{R_{2}, R_{1}-R_{2}\right\} .
$$

Use the $\phi$ above to define the localized time-averaged flux through the shell between the spheres $S\left(\mathbf{x}_{0}, R_{2}\right)$ and $S\left(\mathbf{x}_{0}, R_{1}\right)$ as

$$
\Phi_{\mathbf{x}_{0}, R_{1}, R_{2}}=\frac{1}{T} \iint\left(\frac{1}{2}|\mathbf{u}|^{2}+p\right) \mathbf{u} \cdot \nabla \phi d \mathbf{x} d t .
$$

If $\Phi_{\mathbf{x}_{0}, R_{1}, R_{2}}^{\infty}$ is the anomalous flux inside $A\left(\mathbf{x}_{0}, 2 R_{1}, R_{2} / 2\right)$, i.e., if $\Phi_{\mathbf{x}_{0}, R_{1}, R_{2}}$ satisfies

$$
\begin{aligned}
& \iint\left(\frac{1}{2}|\mathbf{u}|^{2}+p\right) \mathbf{u} \cdot \nabla \phi d \mathbf{x} d t-\Phi_{\mathbf{x}_{0}, R_{1}, R_{2}}^{\infty} \\
& =\nu \iint|\nabla \otimes \mathbf{u}|^{2} \phi d \mathbf{x} d t-\frac{1}{2} \iint|\mathbf{u}|^{2}\left(\partial_{t} \phi+\nu \Delta \phi\right) d \mathbf{x} d t,
\end{aligned}
$$

then define the time average of the modified localized fluxes through the shells $A\left(\mathbf{x}_{0}, R_{1}, R_{2}\right)$ by

$$
\Psi_{\mathbf{x}_{0}, R_{1}, R_{2}}=\Phi_{\mathbf{x}_{0}, R_{1}, R_{2}}-\frac{1}{T} \Phi_{\mathbf{x}_{0}, R_{1}, R_{2}}^{\infty} .
$$

As already mentioned, the modified fluxes can be viewed as total fluxes including the (loss of) flux due to possible singularities inside the shell. Also note that the local energy inequality (2.2) implies

$$
\Phi_{\mathbf{x}_{0}, R_{1}, R_{2}}^{\infty} \geq 0 .
$$


Define the time-averaged energy and enstrophy per unit of mass in the shell between the spheres $S\left(\mathbf{x}_{0}, R_{2}\right)$ and $S\left(\mathbf{x}_{0}, R_{1}\right)$ by

$$
\begin{aligned}
& \mathrm{e}_{\mathbf{x}_{0}, R_{1}, R_{2}}=\frac{1}{T} \iint \frac{1}{2}|\mathbf{u}|^{2} \phi^{2 \delta-1} d \mathbf{x} d t \\
& \mathrm{E}_{\mathbf{x}_{0}, R_{1}, R_{2}}=\frac{1}{T} \iint|\nabla \otimes \mathbf{u}|^{2} \phi d \mathbf{x} d t
\end{aligned}
$$

then,

$$
\tau_{\mathbf{x}_{0}, R_{1}, R_{2}}=\left(\frac{\mathrm{e}_{\mathbf{x}_{0}, R_{1}, R_{2}}}{\mathrm{E}_{\mathbf{x}_{0}, R_{1}, R_{2}}}\right)^{1 / 2}
$$

is the local Taylor length scale associated with the shell $A\left(\mathbf{x}_{0}, R_{1}, R_{2}\right)$.

Note that

$$
\nu|\Delta \phi|=\nu|\eta \Delta \psi| \leq \nu \frac{C_{0}}{\tilde{R}^{2}}\left|\eta \phi^{2 \delta-1}\right| \leq \nu \frac{C_{0}}{\tilde{R}^{2}} \phi^{2 \delta-1}
$$

and

$$
\left|\phi_{t}\right|=\left|\eta_{t} \psi\right| \leq C_{0} \frac{1}{T} \eta^{\delta} \psi \leq \nu \frac{C_{0}}{\tilde{R}^{2}} \phi^{2 \delta-1},
$$

provided

$$
T \geq \frac{R_{0}^{2}}{\nu}\left(\geq \frac{\tilde{R}^{2}}{\nu}\right) .
$$

Hence, (5.4) implies that for any $\mathbf{x}_{0} \in B\left(\mathbf{0}, R_{0}\right)$ and any $0<R_{2}<R_{1} \leq R_{0}$,

$$
\begin{aligned}
\Psi_{\mathbf{x}_{0}, R_{1}, R_{2}} & \geq \nu \mathrm{E}_{\mathbf{x}_{0}, R_{1}, R_{2}}-\nu \frac{C_{0}}{\tilde{R}^{2}} \mathrm{e}_{\mathbf{x}_{0}, R_{1}, R_{2}} \\
& =\nu \mathrm{E}_{\mathbf{x}_{0}, R_{1}, R_{2}}\left(1-C_{0} \frac{\tau_{\mathbf{x}_{0}, R_{1}, R_{2}}^{2}}{\tilde{R}^{2}}\right)
\end{aligned}
$$

leading to the following proposition.

Proposition 5.1. Let $0<\gamma<1$. Then, for any shell $A\left(\mathbf{x}_{0}, R_{1}, R_{2}\right)$ satisfying

$$
\tau_{\mathbf{x}_{0}, R_{1}, R_{2}}<\frac{\gamma}{C_{0}^{1 / 2}} \tilde{R}
$$

with $\tilde{R}$ defined by (5.2),

$$
\Psi_{\mathbf{x}_{0}, R_{1}, R_{2}} \geq \nu \mathrm{E}_{\mathbf{x}_{0}, R_{1}, R_{2}}\left(1-\gamma^{2}\right) .
$$

Similarly, utilizing (5.4) again, we obtain an upper bound

$$
\Psi_{\mathbf{x}_{0}, R_{1}, R_{2}} \leq \nu \mathrm{E}_{\mathbf{x}_{0}, R_{1}, R_{2}}+\nu \frac{C_{0}}{\tilde{R}^{2}} \mathrm{e}_{\mathbf{x}_{0}, R_{1}, R_{2}} .
$$

If the condition (5.13) holds for some $0<\gamma<1$, then it follows that

$$
\Psi_{\mathbf{x}_{0}, R_{1}, R_{2}} \leq \nu \mathrm{E}_{\mathbf{x}_{0}, R_{1}, R_{2}}+\gamma^{2} \nu \mathrm{E}_{\mathbf{x}_{0}, R_{1}, R_{2}} \leq\left(1+\gamma^{2}\right) \nu \mathrm{E}_{\mathbf{x}_{0}, R_{1}, R_{2}} ;
$$

thus, we have arrived at our first locality result. 
Theorem 5.1. Let $0<\gamma<1, \mathbf{x}_{0} \in B\left(\mathbf{0}, R_{0}\right)$ and $0<R_{2}<R_{1} \leq R_{0}$. If

$$
\tau_{\mathbf{x}_{0}, R_{1}, R_{2}}<\frac{\gamma}{C_{0}^{1 / 2}} \tilde{R}
$$

with $\tilde{R}$ defined by (5.2), then

$$
\left(1-\gamma^{2}\right) \nu \mathrm{E}_{\mathbf{x}_{0}, R_{1}, R_{2}} \leq \Psi_{\mathbf{x}_{0}, R_{1}, R_{2}} \leq\left(1+\gamma^{2}\right) \nu \mathrm{E}_{\mathbf{x}_{0}, R_{1}, R_{2}}
$$

where the time average is taken over an interval of time $[0,2 T]$ with $T \geq R_{0}^{2} / \nu$.

Remark 5.1. The theorem states that if the local Taylor scale $\tau_{\mathbf{x}_{0}, R_{1}, R_{2}}$, associated with a shell $A\left(\mathbf{x}_{0}, R_{1}, R_{2}\right)$, is smaller than the thickness of the shell $\tilde{R}$ (a local integral scale), then the time average of the modified flux through that shell towards its center $\mathbf{x}_{0}$ is comparable to the time average of the localized enstrophy in the shell, $\mathrm{E}_{\mathbf{x}_{0}, R_{1}, R_{2}}$. Thus, under the assumption (5.16) the flux through the shell $A\left(\mathrm{x}_{0}, R_{1}, R_{2}\right)$ depends essentially only on the enstrophy contained in the neighborhood of the shell, regardless of what happens at the other scales, making (5.13) a sufficient condition for the locality of the flux through $A\left(\mathbf{x}_{0}, R_{1}, R_{2}\right)$.

Remark 5.2. Similarly as in the case of condition (4.13), we can observe that condition (5.16) can be viewed as a requirement that the time average of a Poincarélike inequality on the shell is not saturating making it plausible in the case of intense fluid activity in a neighborhood of the shell.

Remark 5.3. Since $\Phi_{\mathbf{x}_{0}, R_{1}, R_{2}}^{\infty} \geq 0$, all the lower bounds on the modified fluxes hold for the usual fluxes $\Phi_{\mathbf{x}_{0}, R_{0}, R_{1}}$; in particular, we have

$$
\Phi_{\mathbf{x}_{0}, R_{1}, R_{2}} \geq \nu \mathrm{E}_{\mathbf{x}_{0}, R_{1}, R_{2}}\left(1-C_{0} \frac{\tau_{\mathbf{x}_{0}, R_{1}, R_{2}}^{2}}{\tilde{R}^{2}}\right)
$$

and, provided (5.16) holds,

$$
\Phi_{\mathbf{x}_{0}, R_{0}, R_{1}} \geq\left(1-\gamma^{2}\right) \nu \mathrm{E}_{\mathbf{x}_{0}, R_{1}, R_{2}} .
$$

Also, if a solution $\mathbf{u}$ is such that the (2.2) holds with equality and in particular, if the solution is regular, then all the estimates, including (5.17), hold for the usual averaged flux $\Phi_{\mathbf{x}_{0}, R_{1}, R_{2}}$.

In order to further study the locality of the flux, we will estimate the ensemble averages of the fluxes through the shells $A\left(\mathbf{x}_{i}, 2 R, R\right)$ of thickness $\tilde{R}=R$. Since we are interested in the shells inside $B\left(\mathbf{0}, R_{0}\right)$, we require the lattice points $\mathbf{x}_{i}$ to satisfy

$$
B\left(\mathbf{x}_{i}, R\right) \subset B\left(\mathbf{0}, R_{0}\right) .
$$

To each $A\left(\mathbf{x}_{i}, 2 R, R\right)$ we associate a test function $\phi_{i}=\eta \psi_{i}$ where $\eta$ satisfies (2.5) and $\psi_{i}$ satisfies the following.

If $A\left(\mathbf{x}_{i}, 2 R, R\right) \subset B\left(\mathbf{0}, R_{0}\right)$, then $\psi_{i} \in \mathcal{D}\left(A\left(\mathbf{x}_{i}, 4 R, R / 2\right)\right)$ with

$$
0 \leq \psi_{i} \leq \psi_{0}, \psi_{i}=1 \text { on } A\left(\mathbf{x}_{i}, 2 R, R\right) \cap B\left(\mathbf{0}, R_{0}\right), \frac{\left|\nabla \psi_{i}\right|}{\psi_{i}^{\delta}} \leq \frac{C_{0}}{R}, \frac{\left|\triangle \psi_{i}\right|}{\psi_{i}^{2 \delta-1}} \leq \frac{C_{0}}{R^{2}},
$$

and if $A\left(\mathbf{x}_{i}, 2 R, R\right) \not \subset B\left(\mathbf{0}, R_{0}\right)$ (i.e. we have $B\left(\mathbf{x}_{i}, R\right) \subset B\left(\mathbf{0}, R_{0}\right)$ and $B\left(\mathbf{x}_{i}, 2 R\right) \backslash$ $\left.B\left(\mathbf{0}, R_{0}\right) \neq \emptyset\right)$, then $\psi_{i} \in \mathcal{D}\left(B\left(\mathbf{0}, 2 R_{0}\right)\right)$ with $\psi_{i}=1$ on $A\left(\mathbf{x}_{0}, 2 R, R\right) \cap B\left(\mathbf{0}, R_{0}\right)$ 


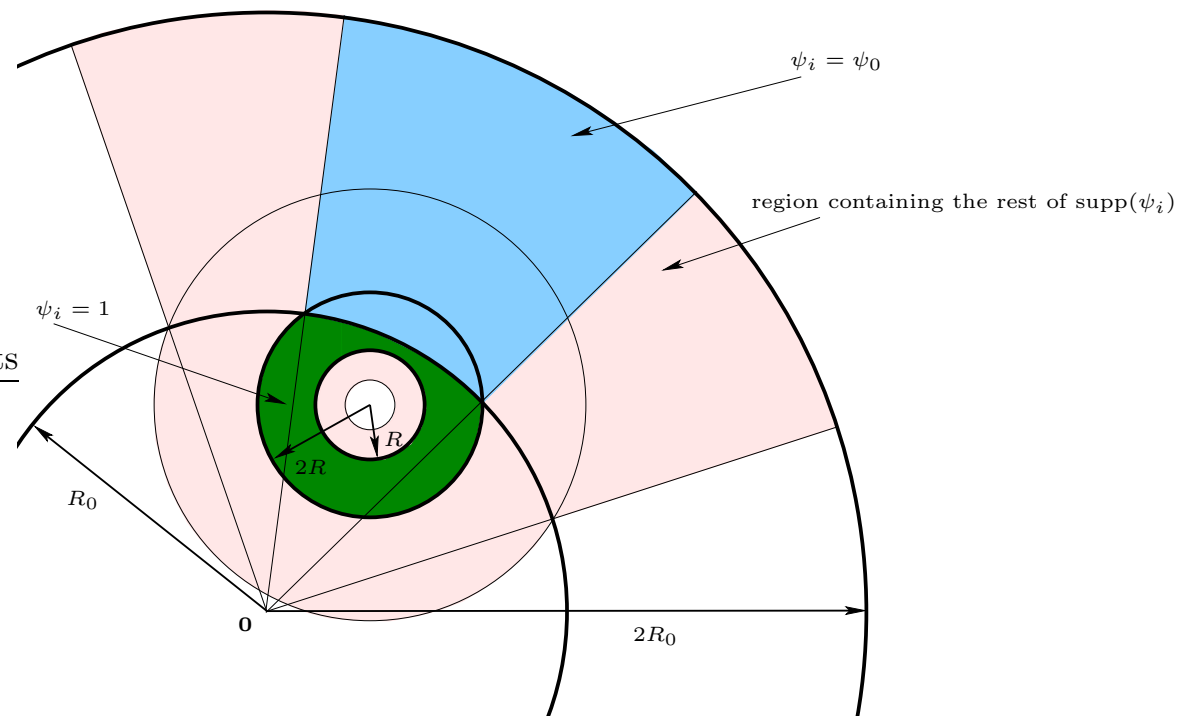

Figure 2. A cross-section of regions of $\operatorname{supp}\left(\psi_{i}\right)$ in the case $A\left(\mathbf{x}_{i}, 2 R, R\right) \not \subset B\left(\mathbf{0}, R_{0}\right)$.

satisfying, in addition to (5.21), the following:

$\psi_{i}=\psi_{0}$ on the part of the cone in $\mathbb{R}^{3}$ centered at zero and passing through $S\left(\mathbf{0}, R_{0}\right) \cap B\left(\mathbf{x}_{i}, 2 R\right)$ between $S\left(\mathbf{0}, R_{0}\right)$ and $S\left(\mathbf{0}, 2 R_{0}\right)$

and

$$
\psi_{i}=0 \text { on } B\left(\mathbf{0}, R_{0}\right) \backslash A\left(\mathbf{x}_{i}, 4 R, R / 2\right) \text { and outside the part of the }
$$

cone in $\mathbb{R}^{3}$ centered at zero and passing through $S\left(\mathbf{0}, R_{0}\right) \cap B\left(\mathbf{x}_{i}, 4 R\right)$

between $S\left(\mathbf{0}, R_{0}\right)$ and $S\left(\mathbf{0}, 2 R_{0}\right)$.

Figure 2 illustrates the definition of $\psi_{i}$ in the case $A\left(\mathbf{x}_{i}, 2 R, R\right)$ is not entirely contained in $B\left(\mathbf{0}, R_{0}\right)$.

Similarly as in the previous section, we consider optimal coverings of $B\left(\mathbf{0}, R_{0}\right)$ by shells $\left\{A\left(\mathbf{x}_{i}, 2 R, R\right)\right\}_{i=1, n}$ such that (5.20) is satisfied,

$$
\left(\frac{R_{0}}{R}\right)^{3} \leq n \leq K_{1}\left(\frac{R_{0}}{R}\right)^{3}
$$

and

$$
\text { any } \mathbf{x} \in B\left(\mathbf{0}, R_{0}\right) \text { is covered by at most } K_{2} \text { shells } A\left(\mathbf{x}_{i}, 4 R, R / 2\right) \text {. }
$$

Introduce

$$
\begin{gathered}
\tilde{\mathrm{e}}_{2 R, R}=\frac{1}{n} \sum_{i=1}^{n} e_{\mathbf{x}_{i}, 2 R, R}, \\
\tilde{\mathrm{E}}_{2 R, R}=\frac{1}{n} \sum_{i=1}^{n} E_{\mathbf{x}_{i}, 2 R, R},
\end{gathered}
$$


and

$$
\tilde{\Phi}_{2 R, R}=\frac{1}{n} \sum_{i=1}^{n} \Phi_{\mathbf{x}_{i}, 2 R, R},
$$

the ensemble averages of the time-averaged energy, enstrophy, and flux on the shells of thickness $R$ corresponding to the covering $\left\{A\left(\mathbf{x}_{i}, 2 R, R\right)\right\}_{i=1, n}$.

The ensemble average of the time-averaged modified flux on shells of thickness $R$ is then defined by

$$
\tilde{\Psi}_{2 R, R}=\frac{1}{n} \sum_{i=1}^{n} \Psi_{\mathbf{x}_{i}, 2 R, R}=\tilde{\Phi}_{2 R, R}-\tilde{\Phi}_{2 R, R}^{\infty}
$$

where

$$
\tilde{\Phi}_{2 R, R}^{\infty}=\frac{1}{n} \sum_{i=1}^{n} \frac{1}{T} \Phi_{\mathbf{x}_{i}, 2 R, R}^{\infty}
$$

is the ensemble average of the time-averaged anomalous fluxes through the shells of thickness $R$ inside $B\left(\mathbf{0}, R_{0}\right)$.

An argument analogous to the one in Lemma 3.1 implies that if the covering of $B\left(\mathbf{0}, R_{0}\right)$ is optimal, then

$$
\tilde{\Phi}_{2 R, R}^{\infty} \leq K \frac{1}{T} \Phi_{\infty} .
$$

Taking the time ensemble averages in (5.4) and applying the bounds (5.9) 5.10$)$, we arrive at

$$
\tilde{\Psi}_{2 R, R} \geq \nu \tilde{\mathrm{E}}_{2 R, R}-\nu \frac{C_{0}}{R^{2}} \tilde{\mathrm{e}}_{2 R, R} .
$$

If the covering is optimal, i.e., if (5.20) and (5.24 (5.25) hold, then

$$
\tilde{\mathrm{E}}_{2 R, R} \geq \frac{1}{n} \tilde{\mathrm{E}} \geq \frac{1}{K_{1}}\left(\frac{R}{R_{0}}\right)^{3} \tilde{\mathrm{E}}
$$

and

$$
\tilde{\mathrm{e}}_{2 R, R} \leq \frac{K_{2}}{n} \tilde{\mathrm{e}} \leq K_{2}\left(\frac{R}{R_{0}}\right)^{3} \tilde{\mathrm{e}},
$$

where

$$
\tilde{\mathrm{E}}=\frac{1}{T} \iint|\nabla \otimes \mathbf{u}|^{2} \phi_{0} d \mathbf{x} d t=R_{0}^{3} \mathrm{E}
$$

is the time average of the localized enstrophy on $B\left(\mathbf{0}, R_{0}\right)$ and

$$
\tilde{\mathrm{e}}=\frac{1}{2} \frac{1}{T} \iint|\mathbf{u}|^{2} \phi_{0}^{2 \delta-1} d \mathbf{x} d t=R_{0}^{3} \mathrm{e}
$$

is the time average of the localized energy on $B\left(\mathbf{0}, R_{0}\right)$ with $\phi_{0}$ is defined by (3.10).

Let us note that

$$
\tau_{0}=\left(\frac{\mathrm{e}}{\mathrm{E}}\right)^{1 / 2}=\left(\frac{\tilde{\mathrm{e}}}{\tilde{\mathrm{E}}}\right)^{1 / 2} .
$$

Utilizing (5.33), (5.34) and (5.37) in the inequality (5.32) gives

$$
\tilde{\Psi}_{2 R, R} \geq \frac{1}{K_{1}}\left(\frac{R}{R_{0}}\right)^{3} \nu \tilde{\mathrm{E}}\left(1-C_{0} K_{1} K_{2} \frac{\tau_{0}^{2}}{R^{2}}\right),
$$


implying the following result.

Proposition 5.2. Assume that the condition 4.13) holds for some $0<\gamma<1$. Then, for any $R$ satisfying (4.15),

$$
c_{0, \gamma}\left(\frac{R}{R_{0}}\right)^{3} \nu \tilde{\mathrm{E}} \leq \tilde{\Psi}_{2 R, R}
$$

holds with $c$ and $c_{0, \gamma}$ defined in (4.14) and 4.17).

Taking the time ensemble averages in the localized energy equality (5.4) again, this time looking for an upper bound, yields

$$
\tilde{\Psi}_{2 R, R} \leq \nu \tilde{\mathrm{E}}_{2 R, R}+\nu \frac{C_{0}}{R^{2}} \tilde{\mathrm{e}}_{2 R, R} .
$$

If the covering $\left\{A\left(\mathbf{x}_{i}, 2 R, R\right)\right\}_{i=1, n}$ of $B\left(\mathbf{0}, R_{0}\right)$ is optimal then, in addition to (5.34),

$$
\tilde{\mathrm{E}}_{2 R, R} \leq \frac{K_{2}}{n} \tilde{\mathrm{E}} \leq K_{2}\left(\frac{R}{R_{0}}\right)^{3} \tilde{\mathrm{E}}
$$

hence,

$$
\tilde{\Psi}_{2 R, R} \leq \nu K_{2}\left(\frac{R}{R_{0}}\right)^{3} \tilde{\mathrm{E}}+\nu K_{2} \frac{C_{0}}{R^{2}}\left(\frac{R}{R_{0}}\right)^{3} \tilde{\mathrm{e}} .
$$

As long as $R$ is inside the inertial range delineated in (4.15),

$$
\tilde{e} \leq c^{2} \gamma^{2} R^{2} \tilde{\mathrm{E}}
$$

leading to

$$
\tilde{\Psi}_{2 R, R} \leq \nu K_{2}\left(\frac{R}{R_{0}}\right)^{3} \tilde{\mathrm{E}}+\nu K_{2} C_{0}\left(\frac{R}{R_{0}}\right)^{3} c^{2} \gamma^{2} \tilde{\mathrm{E}}=c_{1, \gamma}\left(\frac{R}{R_{0}}\right)^{3} \nu \tilde{\mathrm{E}} .
$$

Collecting the bounds on $\tilde{\Psi}_{2 R, R}$ we establish the following.

Theorem 5.2. Assume that the condition 4.13) holds for some $0<\gamma<1$. Then, for any $R$ satisfying $(4.15)$, the ensemble average of the time-averaged modified flux through the shells of thickness $R, \tilde{\Psi}_{2 R, R}$, satisfies

$$
c_{0, \gamma}\left(\frac{R}{R_{0}}\right)^{3} \nu \tilde{\mathrm{E}} \leq \tilde{\Psi}_{2 R, R} \leq c_{1, \gamma}\left(\frac{R}{R_{0}}\right)^{3} \nu \tilde{\mathrm{E}}
$$

where $c, c_{0, \gamma}$, and $c_{1, \gamma}$ are defined in (4.14) and (4.17) and the average is computed over a time interval $[0,2 T]$ with $T \geq R_{0}^{2} / \nu$ and determined by an optimal covering $\left\{A\left(\mathbf{x}_{i}, 2 R, R\right)\right\}_{i=1, n}$ of $B\left(\mathbf{0}, R_{0}\right)$ (i.e. satisfying (5.20), (5.24), and (5.25)).

Note that if

$$
\Psi_{2 R, R}=\frac{1}{R^{3}} \tilde{\Psi}_{2 R, R}
$$

denotes the ensemble average of the time-space averaged modified flux through the shells of thickness $R$ then, dividing (5.41) by $R^{3}$, we obtain the following.

Corollary 5.1. Under the conditions of the previous theorem,

$$
c_{0, \gamma} \nu \mathrm{E} \leq \Psi_{2 R, R} \leq c_{1, \gamma} \nu \mathrm{E} .
$$


Theorem 5.2 allows us to show locality of the time-averaged modified flux under the assumption (4.13). Indeed, the ensemble average of the time-averaged flux through the spheres of radius $R$ satisfying (4.15) is

$$
\tilde{\Psi}_{R}=R^{3} \Psi_{R} .
$$

According to Theorem 4.1,

$$
c_{0, \gamma}\left(\frac{R}{R_{0}}\right)^{3} \nu \tilde{\mathrm{E}} \leq \tilde{\Psi}_{R} \leq c_{1, \gamma}\left(\frac{R}{R_{0}}\right)^{3} \nu \tilde{\mathrm{E}} .
$$

On the other hand, the time ensemble average of the flux through the shells between spheres of radii $R_{2}$ and $2 R_{2}$, according to Theorem 5.2 is

$$
c_{0, \gamma}\left(\frac{R_{2}}{R_{0}}\right)^{3} \nu \tilde{\mathrm{E}} \leq \tilde{\Psi}_{2 R_{2}, R_{2}} \leq c_{1, \gamma}\left(\frac{R_{2}}{R_{0}}\right)^{3} \nu \tilde{\mathrm{E}} .
$$

Consequently,

$$
\frac{c_{0, \gamma}}{c_{1, \gamma}}\left(\frac{R_{2}}{R}\right)^{3} \leq \frac{\tilde{\Psi}_{2 R_{2}, R_{2}}}{\tilde{\Psi}_{R}} \leq \frac{c_{1, \gamma}}{c_{0, \gamma}}\left(\frac{R_{2}}{R}\right)^{3} .
$$

Thus, under the assumption (4.13), throughout the inertial range given by (4.15), the contribution of the shells at scales comparable to $R$ is comparable to the total flux at scales $R$, the contribution of the the shells at scales $R_{2}$ much smaller than $R$ becomes negligible (ultraviolet locality) and the flux through the shells at scales $R_{2}$ much bigger than $R$ becomes substantially bigger and thus essentially uncorrelated to the flux at scales $R$ (infrared locality).

Moreover, if we choose $R_{2}=2^{k} R$ with $k$ an integer, the relation (5.43) becomes

$$
\frac{c_{0, \gamma}}{c_{1, \gamma}} 2^{3 k} \leq \frac{\tilde{\Psi}_{2^{k+1} R, 2^{k} R}}{\tilde{\Psi}_{R}} \leq \frac{c_{1, \gamma}}{c_{0, \gamma}} 2^{3 k}
$$

which implies that the aforementioned manifestations of locality propagate exponentially in the shell number $k$.

In contrast to (5.43), since $\tilde{\mathrm{e}}=R_{0}^{3} \mathrm{e}, \tilde{\mathrm{E}}=R_{0}^{3} \mathrm{E}, \tilde{\Psi}_{2 R_{2}, R_{2}}=R_{2}^{3} \Psi_{2 R_{2}, R_{2}}$ and $\tilde{\Psi}_{R}=R^{3} \Psi_{R}$

$$
\frac{c_{0, \gamma}}{c_{1, \gamma}} \leq \frac{\Psi_{2 R_{2}, R_{2}}}{\Psi_{R}} \leq \frac{c_{1, \gamma}}{c_{0, \gamma}}
$$

i.e., the ensemble averages of the time-space averaged modified fluxes of the flows satisfying (4.13) are comparable throughout the scales involved in the inertial range (4.15) which is consistent with the universality of the cascade.

We conclude this section by noticing that the remarks similar to those at the end of section 4 can be applied here. Namely we have the following.

Remark 5.4. Since $\tilde{\Phi}_{2 R, R}^{\infty} \geq 0$, then all the lower bounds on modified fluxes hold for the usual fluxes $\tilde{\Phi}_{2 R, R}$ and $\Phi_{2 R, R}$; in particular, we have

$$
\tilde{\Phi}_{2 R, R} \geq c_{1}\left(\frac{R}{R_{0}}\right)^{3} \nu \tilde{\mathrm{E}}\left(1-c_{2} \frac{\tau_{0}^{2}}{R^{2}}\right)
$$

and, provided (4.13) and (4.15) hold,

$$
\tilde{\Phi}_{2 R, R} \geq c_{0, \gamma}\left(\frac{R}{R_{0}}\right)^{3} \nu \tilde{\mathrm{E}}
$$


Also, if a solution $\mathbf{u}$ is such that (2.2) holds with equality and in particular, if the solution is regular, then all the estimates, including (5.41) and (5.42), hold for the usual averaged fluxes $\tilde{\Phi}_{2 R, R}$ and $\Phi_{2 R, R}=\tilde{\Phi}_{2 R, R} / R^{3}$.

Remark 5.5. If the additional assumptions (5.22) and (5.23) for the test functions on the shells $A\left(\mathbf{x}_{i}, 2 R, R\right)$ which are not contained entirely in $B\left(\mathbf{0}, R_{0}\right)$ are not imposed, then the lower bounds in (5.38) and (5.41) hold with $\tilde{E}$ replaced by the time average of the non-localized in space enstrophy on $B\left(\mathbf{0}, R_{0}\right)$,

$$
\tilde{E}^{\prime}=\frac{1}{T} \int_{0}^{2 T} \int_{B\left(\mathbf{0}, R_{0}\right)}|\nabla \otimes \mathbf{u}|^{2} \eta d \mathbf{x} d t=R_{0}^{3} E^{\prime} .
$$

This is the case because the estimate (5.33) gets replaced with

$$
\tilde{\mathrm{E}}_{2 R, R} \geq \frac{1}{K_{1}}\left(\frac{R}{R_{0}}\right)^{3} \tilde{E}^{\prime}
$$

Also, the estimates (5.43) and (5.45) will contain the terms $E^{\prime} / \mathrm{E}\left(=\tilde{E}^{\prime} / \tilde{\mathrm{E}}\right)$ in the lower and $\mathrm{E} / E^{\prime}$ in the upper bounds.

\section{REFERENCES}

[1] L. Caffarelli, R. Kohn, and L. Nirenberg. Partial regularity of suitable weak solutions of the Navier-Stokes equations. Comm. Pure Appl. Math., 35(6):771-831, 1982.

[2] A. Cheskidov, P. Constantin, S. Friedlander, and R. Shvydkoy. Energy conservation and Onsager's conjecture for the Euler equations. Nonlinearity, 21(6):1233-1252, 2008.

[3] P. Constantin, W. E, and E. Titi. Onsager's conjecture on the energy conservation for solutions of Euler's equations. Comm. Math. Phys., 165(1):207-209, 1994.

[4] P. Constantin and C. Foias. Navier-Stokes equations. Chicago Lectures in Mathematics. University of Chicago Press, Chicago, IL, 1988.

[5] G. L. Eyink. Locality of turbulent cascades. Phys. D, 207(1-2):91-116, 2005.

[6] G. L. Eyink and K. R. Sreenivasan. Onsager and the theory of hydrodynamic turbulence. Rev. Mod. Phys., 78(1):87-135, 2006.

[7] C. Foias, O. Manley, R. Rosa, and R. Temam. Navier-Stokes equations and turbulence, volume 83 of Encyclopedia of Mathematics and its Applications. Cambridge University Press, Cambridge, 2001.

[8] C. Foias, O. P. Manley, R. M. S. Rosa, and R. Temam. Estimates for the energy cascade in three-dimensional turbulent flows. C. R. Acad. Sci. Paris Sér. I Math., 333(5):499-504, 2001.

[9] U. Frisch. Turbulence. Cambridge University Press, Cambridge, 1995. The legacy of A. N. Kolmogorov.

[10] A. N. Kolmogorov. Dissipation of energy in the locally isotropic turbulence. Dokl. Akad. Nauk SSSR, 32:16-18, 1941.

[11] A. N. Kolmogorov. The local structure of turbulence in incompressible viscous fluid for very large Reynolds numbers. Dokl. Akad. Nauk SSSR, 30:9-13, 1941.

[12] A. N. Kolmogorov. On generation of isotropic turbulence in an incompressible viscous liquid. Dokl. Akad. Nauk SSSR, 31:538-540, 1941.

[13] R.H. Kraichnan. Inertial-range transfer in two- and three-dimensional turbulence. J, Fluid Mech., 47:525-535, 1971.

[14] P. G. Lemarié-Rieusset. Recent developments in the Navier-Stokes problem, volume 431 of Chapman \& Hall/CRC Research Notes in Mathematics. Chapman \& Hall/CRC, Boca Raton, FL, 2002.

[15] V. L'vov and G. Falkovich. Counterbalanced interaction locality of developed hydrodynamic turbulence. Phys. Rev. A, 46(8):4762-4772, 1992.

[16] L. Onsager. Statistical hydrodynamics. Nuovo Cimento (9), 6(Supplemento, 2(Convegno Internazionale di Meccanica Statistica)):279-287, 1949. 
[17] V. Scheffer. Hausdorff measure and the Navier-Stokes equations. Comm. Math. Phys., 55(2):97-112, 1977.

[18] R. Temam. Navier-Stokes equations. AMS Chelsea Publishing, Providence, RI, 2001. Theory and numerical analysis, Reprint of the 1984 edition.

Department of Mathematics, University of Virginia, Charlottesville, VA 22904

Department of Mathematics, University of Virginia, Charlottesville, VA 22904 